\title{
Automated Tool for Diagnosis of Sinus Analysis CT Scans
}

\author{
Abdel-Razzak Natsheh ${ }^{1}$, Prasad VS Ponnapalli ${ }^{1}$, Nader Anani ${ }^{1}$, \\ Atef El-Kholy ${ }^{2}$ \\ 'Department of Engineering and Technology, Manchester Metropolitan \\ University, Manchester M1 5GD \\ ${ }_{2}^{2}$ Trafford General Hospital, Manchester M41 5SL
}

\begin{abstract}
Diagnosis of Sinus condition is considered a difficult task in medical clinics due to the similar nature of the symptoms and the complexity of the images (e.g. plane of image, resolution) obtained using either CT-Scan. Discussions with consultant doctors and radiologists working in this area pointed at the need for a computer-based analysis and diagnosis tool that could be used as an aid to experts for diagnosing sinus diseases. There are a number of tools using traditional image processing techniques that are primarily useful for enhancing images. For an integrated system with potential diagnostic abilities artificial neural networks are good candidates that can combine image processing and diagnostic abilities in a single system. This paper presents the background and preliminary results in the development of an automated tool for the analysis and diagnosis of sinus conditions. The data used is in the form of CT scan images of sinus. Technology based on traditional image processing and Artificial Neural Networks (SOM) are explored for image processing and diagnosis. Anonymous CT-images of Sinuses were obtained from a local hospital. Preliminary results show that the proposed system has the potential to be a useful tool for clinicians in the areas of diagnosis and training of junior doctors.
\end{abstract}

\section{Introduction}

Diagnosis of a Sinus condition is a process that involves the use of multiple sources of information (e.g. laboratory and visual tests, or CT-scan images) and expertise of a clinician in interpreting the images. In addition to the commonly used clinical information, one of the key features of Sinus condition diagnosis is the quality and complexity of the images that a clinician uses to make decisions. 
The images can differ considerably in terms of quality, angles of imaging while the analysis can be further complicated by the irregularity of patients' anatomy, the extent of diseased sinus area and bone structure. Considering the similarity of symptoms for a range of underlying problems, an ENT doctor has to use multiple threads of reasoning employing qualitative and quantitative measures to arrive at a diagnosis. Such decisions are harder to make when the related aetiology is hard to discern or when multiple diseases are suffered [1]. Discussions with consultant doctors and radiologists working in this area pointed at the need for a computerbased analysis and diagnosis tool that could be used not only as an aid to experts but also for use in the training of doctors in this area.

Previous work reported potential benefits of using ANNs in enhancing diagnosis and early intervention diagnosis [2]. However, a review of literature showed that the capabilities of ANNs have not been exploited for diagnosis in the sinus field. In particular, diagnosis and classification of disease in sinus is done using a variety of image-based information (e.g. CT scans). This project is aimed at developing and validating ANN-based techniques to help diagnosis in this area by using novel methods of integrating image processing, estimation of quantitative measures and diagnosis stages into a unified system.

The proposed system combines artificial neural networks (Self Organising Maps (SOM) [3]) and the use of feature extraction methods for giving the user a potential set of diagnoses. The results are given in the form of opacity percentage of the sinus areas referred to as Region of Interest (ROI) in this work.

The rest of paper is organised as follows. Section 2 presents a general look on sinus anatomy and sinus imaging. Section 3 presents the architecture of the hybrid system, the use of feature extraction techniques to identify the ROI and the use of SOMs in classifying the extracted data to get the diagnosis result (opacity percentage). Section 4 presents the results of the application to a set of CT-scan images and the conclusions are presented in Section 5.

\section{Sinus Diagnosis and Medical Imaging}

A brief introduction to the terminology of the sinus area is presented here to explain the imaging and diagnosis problems. The Paranasal sinus system comprises eight (four pairs) air-filled spaces, or sinuses, within the bones of the skull and face. These are divided into subgroups that are named according to which bones they lie under.

- The maxillary sinuses, also called the antra, are under the eyes in the maxillary bones (cheek bones).

- The frontal sinuses lie over the eyes, in the frontal bone which forms the hard part of the forehead.

- The ethomoid sinus, between the nose and the eyes, extends backwards into the skull. 
- The sphenoid sinuses lie in the centre of the skull base.

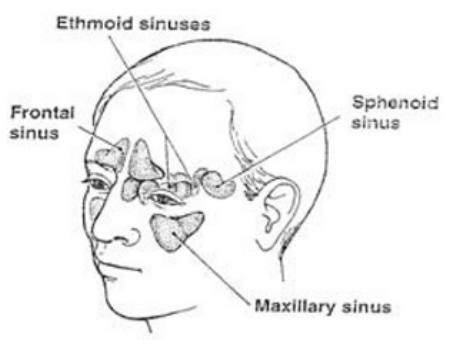

Figure 1: Normal Sinus Anatomy [8]

The sinus groups described above constitute the ROI in this work. Identification of this region (together with the immediate bone structure), analysis of opacity of the region and diagnosis (classification) of opacity form the main objectives this research. Inflammatory sinus disease is a serious health issue that can affect anyone. Accurate and timely diagnosis is of primary importance in the management of sinus diseases for patients referred with sinusitis symptoms. CTscans are one of the most widely used imaging tools in the diagnosis of sinus diseases. One of the important features that make CT-images the number one choice for surgeons to use in sinus diagnosis is that they provide a view of the nasal and paranasal sinuses, which are not obtainable by any other imaging modality, in order for otolaryngologists to make an accurate diagnosis and develop an effective treatment plan. In addition to being a valuable diagnostic tool, coronal CT scans also serve as a surgical "roadmap" for the surgeon performing FESS (Functional Endoscopic Sinus Surgery) [1].

\section{Architecture of the Sinus Diagnostic System}

The architecture of the proposed complete automated diagnostic system is shown in Figure 2. The system uses the CT images of sinus as inputs. It contains three main modules to perform the three major functions of the diagnostic process: (i) Extraction of ROI, (ii) Classification; and (iii) Opacity measures and Diagnosis. The implementation strategy is to develop each of the prototype modules and refine them iteratively using more complex images and disease conditions. Details of each stage are discussed in the following sections. All the implementation is done in the Simulink/Matlab ${ }^{\mathrm{TM}}$ environment. 


\section{Architecture of the Sinus Diagnostic System (SDS)}

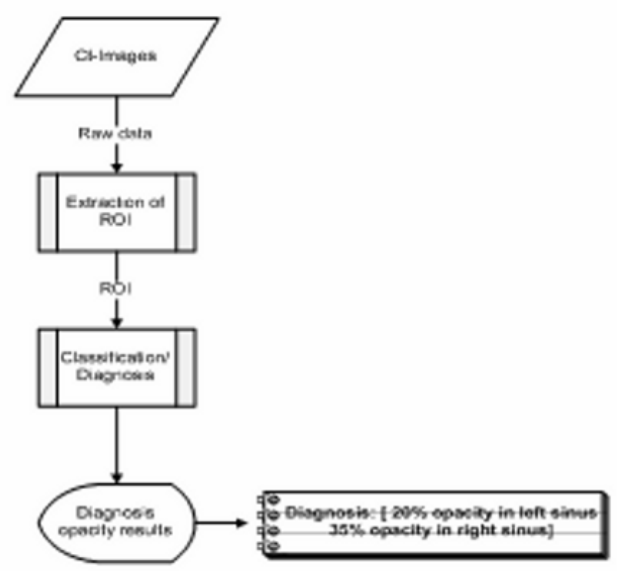

Figure 2: Development stages for the Sinus Diagnosis System

\subsection{Sinus CT Feature Analysis}

For an otolaryngologist CT image scans are an important tool for sinus diagnosis. Figure 5 shows an example of sinus CT- images of a patient in different angular positions. In this work $33 \mathrm{CT}$ images taken from different angles for each patient are used in the initial analysis. Each CT image has a resolution of 1012x938 pixels.

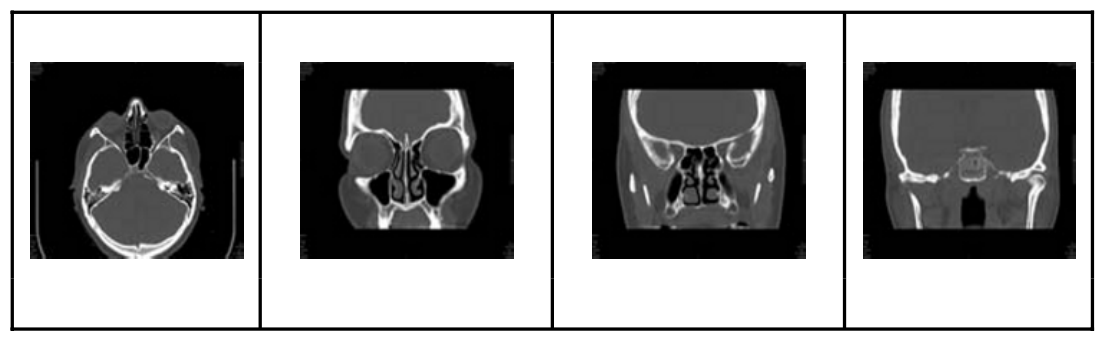

Figure 3: CT-Scans of a typical sinus in different anglular positions

Image analysis work has been carried out in order to extract the main features of the sinus CT-images. The important features are discussed in the following.

(1) CT images can be described as grey scale images; each image consists of a number of pixels with grey scale (intensity) values in the range of [0$255]$ or if normalised $[0-1]$. Such values are used in classifying and labelling different vital organs in medical imaging. Table 1 shows sinus classes and their details in terms of intensity values. 


\begin{tabular}{|l|l|}
\hline Sinus Classes & Intensity values \\
\hline Black (Background, Air) & -1000 \\
\hline White (bone) & $(+600$ to +1000$)$ \\
\hline Grey (tissue) & $(+30$ to $60+)$ \\
\hline Muscle & +73 \\
\hline Optic nerve & +70 \\
\hline
\end{tabular}

Table 1: shows sinus classes and their details regarding intensity values

(2) Multi-Scale feature is one of the important and noticeable features that make analysis of sinus CT-images difficult. Multi-Scaling means the images can have scale variation in shape, size, and angle. Different patients have different skull-bone structures, which mean they have different anatomy and structure of sinuses.

(3) In sinus CT, different sinus types are displayed in different CT sections due to the positioning of sinus types [1]; therefore image analysis work has to be carried out on all of the different CT sections (different angular positions) in a typical CT-image set in order to examine the whole of the sinus area.

\subsection{Extraction of ROI}

The ROI in this work is defined as the actual sinus area within a CT image (cf. Figure 4, cf. Figure 1). In this work, having CT images (input data) with high resolution is considered one of the factors which presents the extraction of ROI as a good alternative solution to the intensive cost of computational process time if the whole image were to be analysed. When performing analysis of complex data, one of the major problems arises from the multiplicity of data points involved. Analysis with a large number of data points generally requires large amounts of memory and computation power. Feature extraction is a general term for methods of constructing combinations of the data/variables to get around these problems while still describing the data with sufficient accuracy [4].

Adding to the above mentioned problems, classifying the whole CT image rather than a specified area (ROI) increases the error percentage value in terms of diagnosis accuracy. Hence extraction of ROI (i.e. feature extraction) is a vital stage in the sinus diagnosis problem. The main steps in the current implementation of the ROI extraction stage are shown in Figure 5 below. 


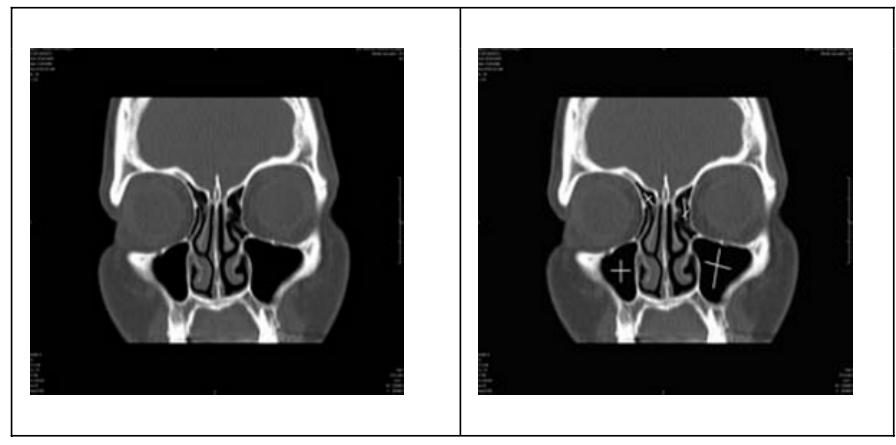

Figure 4: Regions of Interest from a section of a CT scan

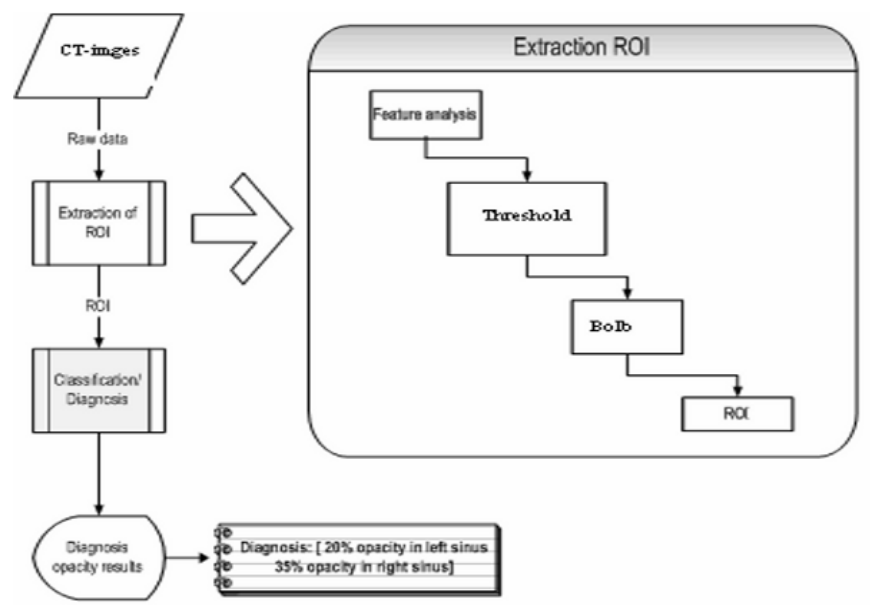

Figure 5: Stages in the extraction of the Region of Interest

\subsubsection{CT image pre-processing}

Image pre-processing phase is an essential phase in medical imaging [4]. CT images have blurred edges which need to be processed so that they are well defined. In such cases filtering methods are applied to CT images for smoothing, sharpening, and enhancing images edges.

Image analysis work is carried out in this stage, starting with investigating the CT image using histogram. CT histogram (cf. Figure 6) explains the image structure in terms of pixel intensity value distribution (pixel value 0 means black, 1 white, and in between these values 0-1 means grey). Finding the peak point in the histogram means finding the intensity value which most of the image pixels have which can be used in the threshold method. 


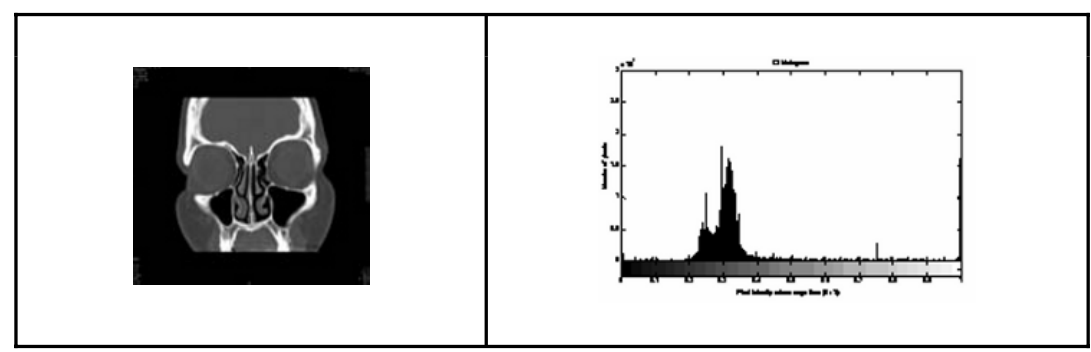

Figure 6: Histogram of a filtered Sinus image

The next step is to convert the whole CT image from grey scale format into binary format just (0-1), as shown in Figure 7. This conversion from grey to binary image provides the outline of the bone structure within the image which in turn will be used to identifying and labelling the ROI of sinus.

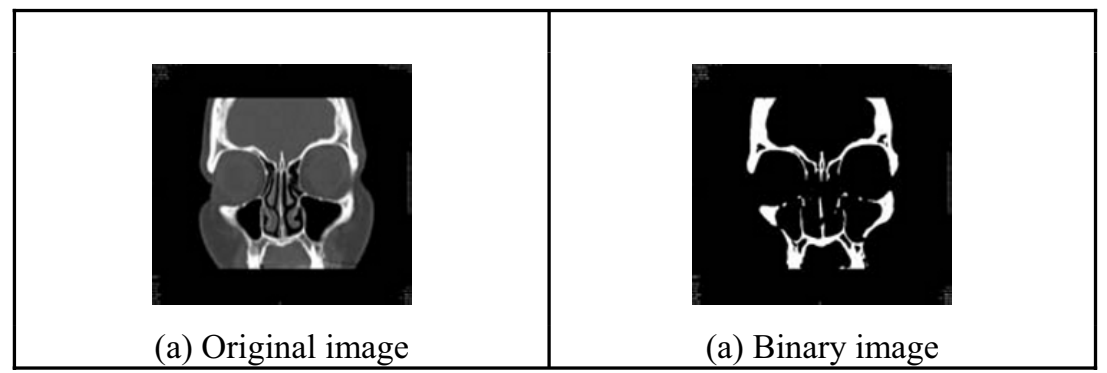

Figure 7: Sinus images: Original and binary image format

\subsubsection{Threshold phase}

The binary image provides the bone structure information. However, the extraction of ROI is still a difficult problem as commonly used techniques such as template matching cannot be used due to the high variability in the sinus image area because of the angle of CT scan and the variance in the shapes from one individual to the other. In typical image processing applications threshold method is used to segment CT images depending on intensity values [7]. In this application, the thresholding method is used to complete the shape boundaries so that the CT image can be converted to an image consisting of closed objects (contours). This is then used to identify the sinus ROI.

The peak value provided by histogram of the image pixels is used as a threshold value. Each pixel that has less intensity value than the threshold value is converted into 0 values (black); otherwise the pixel value is converted to white (binary format display). Using the 'right' threshold value this method helped in generating an image consisting of closed shapes (contours) that represent the bone structure surrounding a sinus area (cf. Figure 9). Finally for labelling and identifying the ROI for sinus, the following transformation is applied to the Thresholded CT image (TCT). 


$$
R O I=1-T C T
$$

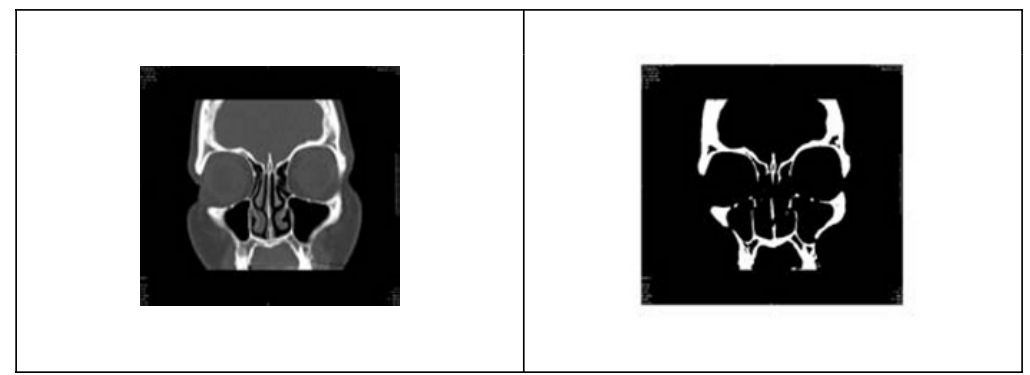

Figure 8: Illustration of image processing problem (incomplete shapes)

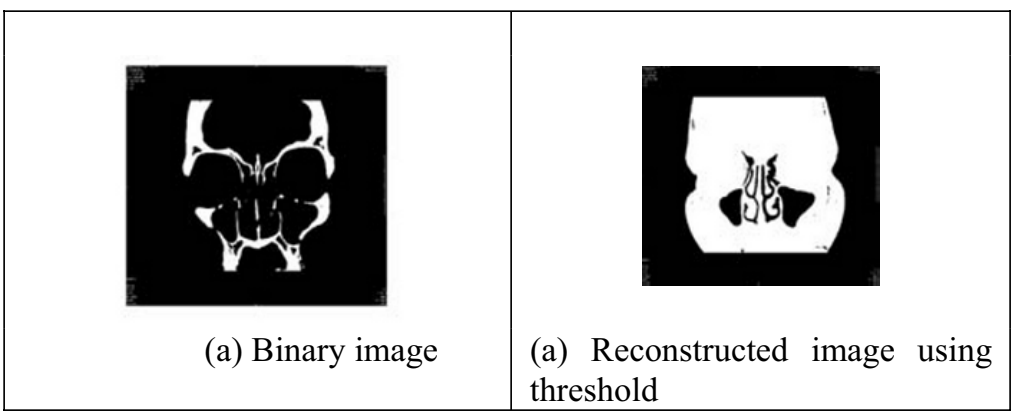

Figure 9: Image processing using the threshold method

\subsubsection{Blob analysis}

The thresholding and transformation phase described in the previous section resulted in an image with closed contours. The next step is to extract as much information as possible from the ROI corresponding to the sinus areas within the image. This stage is rather obvious for a clinician but is far more challenging for an automated system for reasons already discussed. In the present work blob analysis is used to extract main features of the ROIs.

A group of pixels organized into a structure is commonly called a blob. In this phase, three ROI areas (maxillary, ethmoid, frontal sinus areas) are labelled as blob structures.

Blob analysis methods were applied on the labelled objects; to get region properties for each object. Depending on the Area size property for each object, ROI areas was extracted. Fig.10 shows the CT-image after applying blob analysis. 


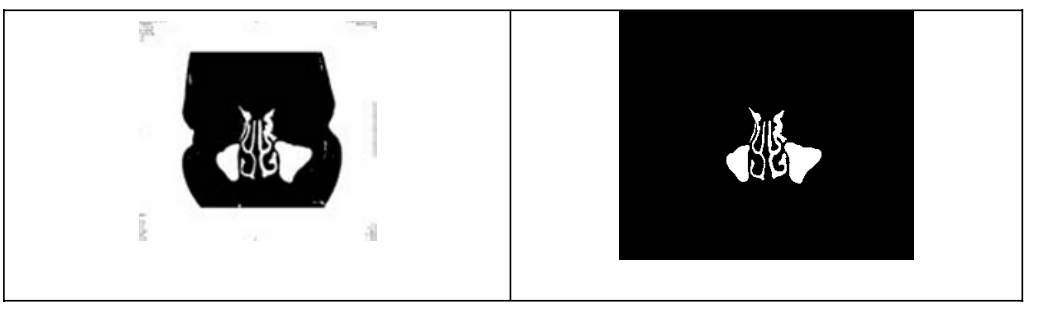

Figure 10: Blob Analysis: Image after applying threshold method

\subsection{Classification and Diagnosis}

The final phase corresponds to the main aim of the work, i.e. to classify each $R O I$ into three regions corresponding to air (Black), bone (White) and soft tissue (Grey). The percentage of grey region is to be used as an indicator (quantitative measure) of the extent of 'disease' in the sinus area. A number of methods exist to perform such classification.

In image classification field, unsupervised neural networks such as SOM have proven to have more robust and accurate than supervised neural networks. Ahmed and Farag (1998) used two self-organizing maps (SOM) in two stages, selforganizing principal components analysis (SOPCA) and self-organizing feature map (SOFM), for automatic volume segmentation of medical images [25]. They performed a statistical comparison of the performance of the SOFM with Hopfield network and ISODATA algorithm. The results indicate that the accuracy of SOFM is superior to that obtained by the other two networks. In addition, SOFM was claimed to have the advantage of ease implementation and guaranteed convergence.

For the initial studies, two classification methods were applied to the (input data in the ROI shown in Table 2): Threshold method and Self Organizing Map-based classification (cf. Figure 11). The minimum and maximum values that can occur for the pixels are 0 (Black) and 255 (White). These are scaled to the range of [01] for calculating opacity. Normal sinus areas tend to have an average opacity value of close to zero.

SOM algorithm has been chosen over the threshold method, because it is robust and efficient in multidimensional classification. Compared with traditional statistical classifiers, such as the threshold method, neural network methods perform relatively well due to their exceptional ability of generalization. Selforganizing map classifiers have been widely used in medical image segmentation.

There is also great potential for combining the classical segmentation methods with the neural network algorithms to greatly improve the performance of image segmentation. 


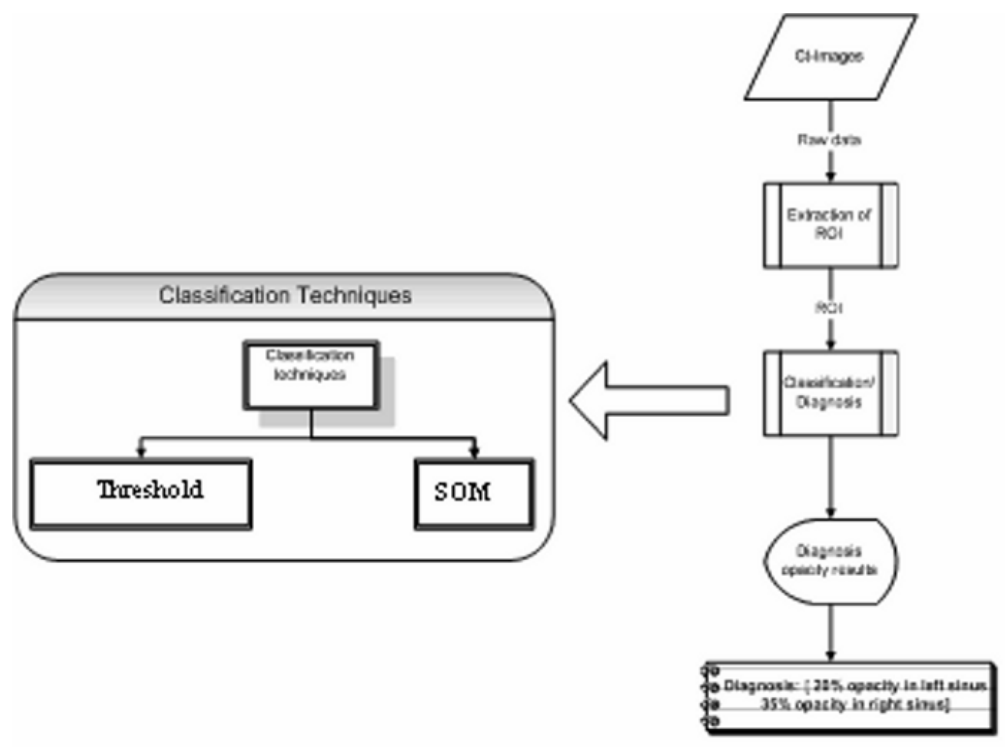

Figure 11: Classification techniques

\begin{tabular}{|l|c|c|}
\hline \multicolumn{1}{|c|}{ Image name } & Cropped image & Binary mask ROI \\
\hline $\begin{array}{l}\text { Left maxillary sinus } \\
\text { area (LMXR) }\end{array}$ & \\
\hline $\begin{array}{l}\text { Right maxillary } \\
\text { sinus area (RMXR) }\end{array}$ & \\
\hline $\begin{array}{l}\text { Infected maxillary } \\
\text { sinus area (infected } \\
\text { LMXR) }\end{array}$ & \\
\hline
\end{tabular}

Table 2: Cropped sinus images and their binary images with the extracted ROI 


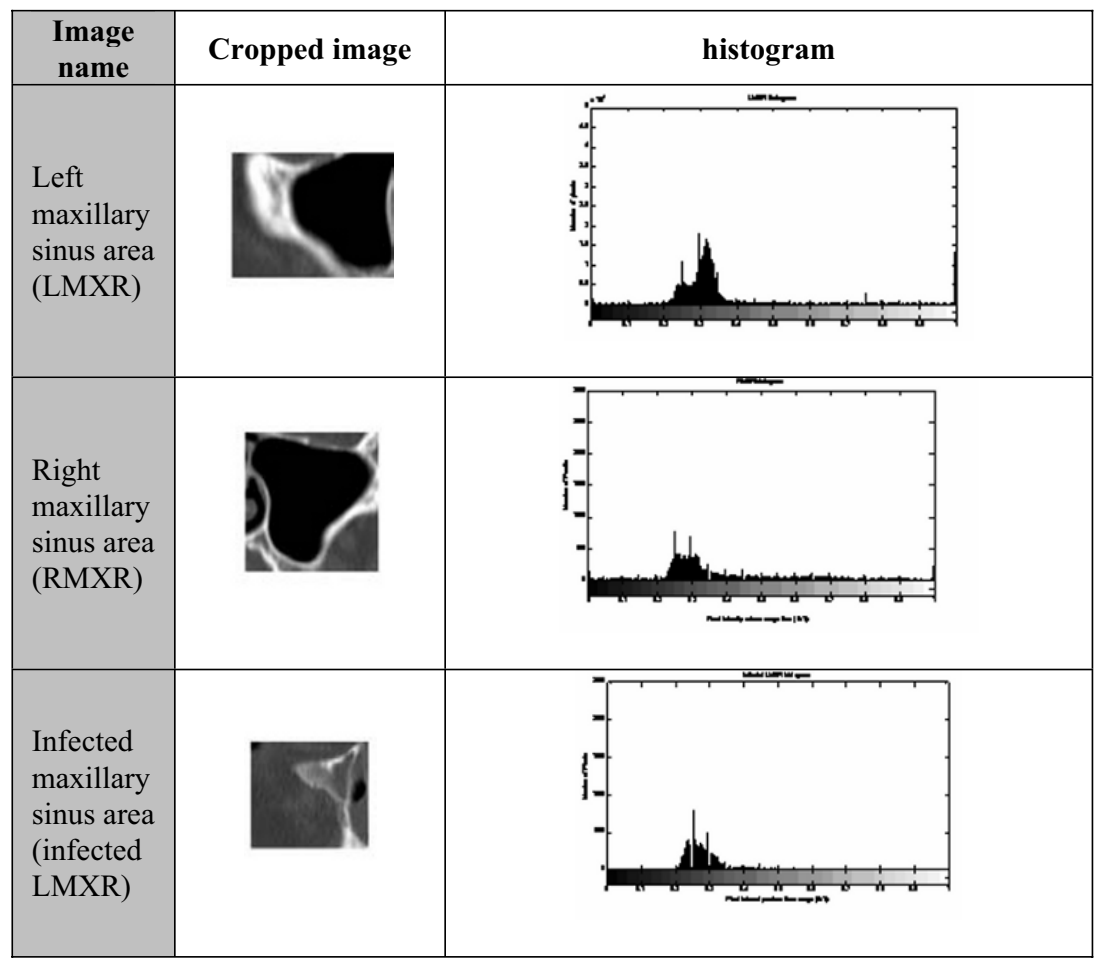

Table 3: Cropped sinus images and their binary images with the extracted ROI

\subsubsection{Neural Network Training issues}

As the objective was to classify the ROI into air, grey and bone regions, a SOM with 3 outputs (classes) hextop topology grid was created. The SOM was provided all the ROI pixel values as inputs. It was then trained to classify all the values into 3 classes. The corresponding centroids for these classes were represented by the weights in the $S O M$. Once trained, the $S O M$ was then used on the sinus area only for classifying the grey level intensity values.Initially the $S O M$ Neural Network was trained for 300 iterations. The resulting weights after training are shown in Table 4. The classification of the SOM network has to be interpreted in terms of the physical phenomena they represent. This is shown in Table 4.

\begin{tabular}{|l|l|}
\hline Class & Pixel intensity range \\
\hline Class 1 (Air) & 0.0356 \\
\hline Class 2 (Grey) & 0.2840 \\
\hline Class 3 (Bone) & 0.6935 \\
\hline
\end{tabular}

Table 4: Centroids of SOM network classes 


\subsubsection{Threshold method}

Based on the Grey level histograms shown in Table 3, a set of threshold values shown in Table 5 were chosen heuristically as a basis to identifying the opacity and thus classify the sinus ROI area into 3 classes.

\begin{tabular}{|l|l|}
\hline Class & Pixel intensity range \\
\hline Black (Air) & $<0.25$ \\
\hline Gray (Grey) & $0.25-0.56$ \\
\hline White (Bone) & $>0.56$ \\
\hline
\end{tabular}

Table 5: threshold values based on the histograms shown in Table 3

For each class, the total count of pixels corresponding to that class was calculated and was assumed to represent the "Class Area" $(C A)$ of that class in the total image. The Percentage Area $(P A)$ for each class was then calculated as:

$$
P A\left(C_{i}\right)=\frac{C A\left(C_{i}\right)}{T A}=\frac{\left(\text { number_of_pixel_ } C_{i}\right)}{\text { Total_number_of_pixels }}
$$

In this particular case, Total Area (TA), as represented by pixel count, was 22084 for the ROI.

\section{Comparative Results: ROI}

One set of results of the classification achieved using the two methods, SOM and Threshold, is shown in Table 6 below. The image with the largest variations (Image 2 in Table 6) was used to extract the threshold values and also to train the SOM for classification into three classes. The resulting threshold values and SOM were used to classify the other two images. The classification results of the first two healthy images were comparable in both cases. The third image, which represents a sinus with disease, provided a test case for the efficacy of the two approaches. Grey intensity pixel values in medical images can be ambiguous in terms of interpretation. They could represent an area of diseased tissues or muscle, or could be just noise in the image due to error in positioning. In this matter SOM is very powerful tool in differentiating between the diseased tissues or muscle for example. This is because it has the capability of processing and learning from a large amount of information rich data as an input which enables in learning more features of the data.

The grey intensity pixels values are different from one image to another, and getting a fixed threshold value to get the grey opacity by the threshold method is difficult; SOM is easier to implement and it is a more dynamic approach compared to the threshold method. More than one image can be provided as an input to SOM which helps in the learning of threshold values that will fit most of the cases, thus enabling it to classify more accurately than the threshold method. (eg. 5 th and last image in table 6). Developing a measure to quantify the accuracy is an area currently under investigation. However, the 'accuracy' of the two classifications 
indicated that the results obtained using the SOM were closer to a typical clinician's diagnosis.

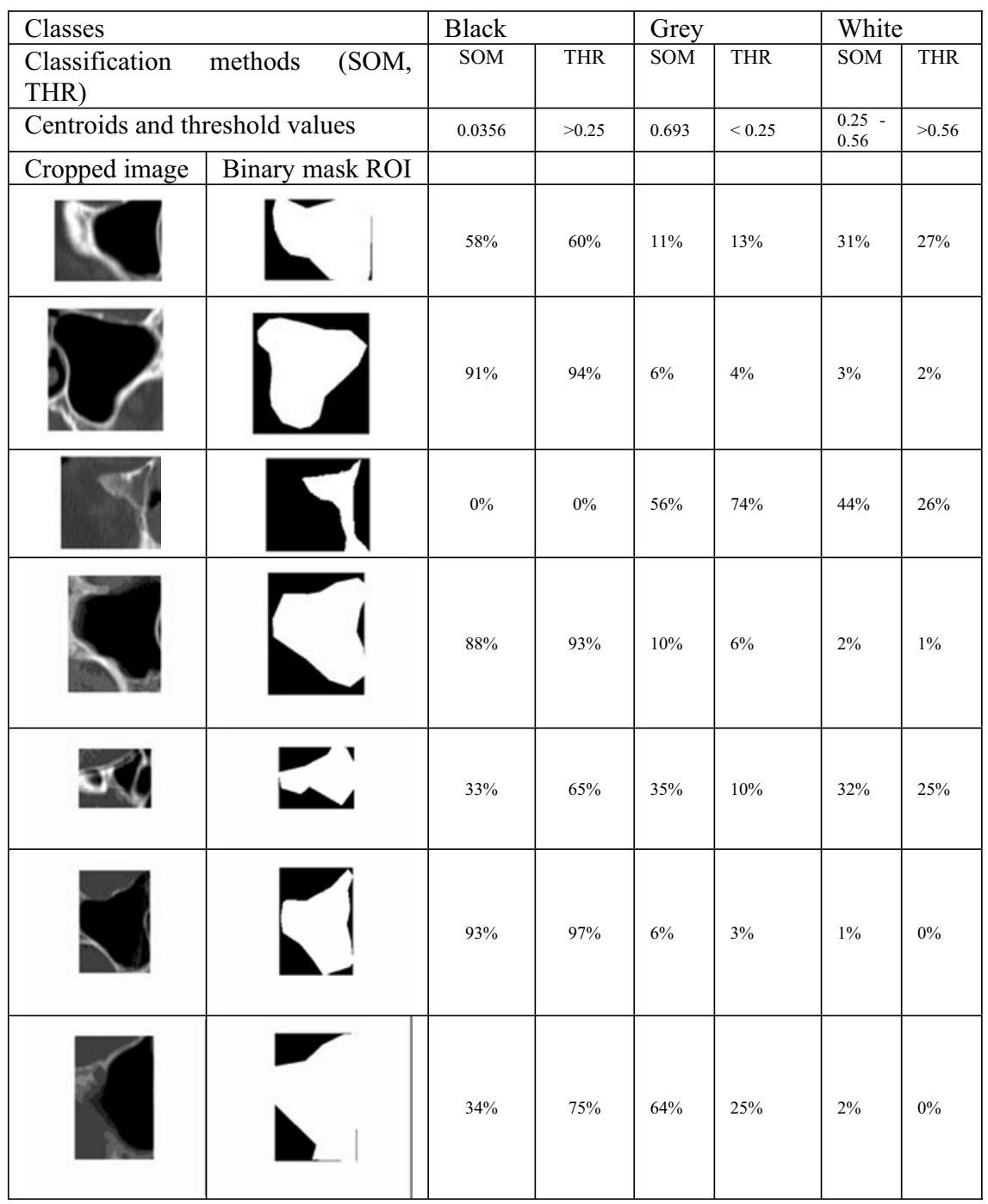

Table 6: Comparison between the two classification methods

\section{Discussion and Conclusion}

This work presents a new NN-based algorithm for diagnosing Sinus diseases, based on the opacity percentage that is useful for diagnosing sinus cases. Notice that both of the output results shown in Table 6 have a reasonable opacity percentage representation of the sinus images, with a slight variation.

Initial consultation with experts in the field suggested a good degree of confidence in the classification of the opacity of the sinus areas. However, considerable testing 
of the system is still required to arrive at a measure of the degree of confidence of the decisions that can be made based on the results. The actual diagnosis part that will link the quantitative measures (together with a knowledge base) to a possible disease is still under development.

Further work is in progress using $S O M$ to extract and classify the $R O I$ in to two classes black and grey. Work is also under progress to validate the results using a large set of data and to develop and customise the SOM-based algorithm for good generalisation. Finally, this approach will be very helpful and important for practising and consulting doctors, by providing them with the actual regions of interest and quantitative measures (such as percentage opacity) thus help reduce critical time in decision making. Further, it will be a very useful tool for training doctors for use as a help system in evaluating their clinical decisions.

\section{References}

1. Kennedy D.W, Bolger William E, Zinreich S.James. Diseases of Sinuses Diagnosis and Management. B.C. Decker Inc. 2000.

2. Lisboa, P.J.G. A review of evidence of health benefit from artificial neural networks in medical intervention. Neural Networks, 2002. 15, p. 11-39.

3. Kohonen T, The Self-Organizing Map. vol. 78(9). Proceedings of the IEEE, 1990. p. 1464-1480.

4. Kass M, Witkin A, Terzopoulos, D. Snakes: Active contour models. International Journal of Computer Vision, 1988. 1(4):321- 331.

5. Xuan J, Addli T, Wang Y, "Segmentation of magnetic resonance brain image: integrating region growing and edge det ec tion," IEEE Proceedings of International Conference on Image Processang, (Washington, DC). 1995., vol. 3, pp.544-547.

6. Kamber M, Shinghal R, Collins L, Francis G.S,"Model based 3-D segmentation of multiple sclerosis lesions in magnetic resonance brain images," IEEE Trans. Med. Image. 1995., vol. 14, pp. 442-453.

7. Pohle Regina, D. Toennies Klaus. Segmetation of Medical Images using Adaptive Region Growing, Proc. SPIE 2001, Vol. 4322, pp. 1337-1346.

8. http://cfcenter.stanford.edu/sinus-graphic.gif 